\title{
Detection of periodically and randomly interrupted noise signals*
}

\author{
RICHARD E. PASTORE $\dagger$ \\ Central Institute for the Deaf, St. Louis, Missouri 63110
}

\begin{abstract}
The detectability of a masked, interrupted, band-limited $(150-2,400 \mathrm{~Hz})$ noise signal was studied as a function of whether the interruption process was periodic or' random. A standard monaural two-interval, temporal forced-choice detection procedure was employed both with and without a low-level contralateral cue (MDCC). The cue was an independent noise interrupted synchronously with the signal. Detection performance was equal under all conditions and was equivalent to the detection of an uninterrupted noise signal of equal power. Furthermore, like speech waveforms, the two types of interruption processes were discriminable only at levels at least $10-12 \mathrm{~dB}$ above the level required for detection.
\end{abstract}

The change or interruption of a stimulus is probably at least as significant to an organism as the simple continuation of that stimulus. The onset and offset of stimuli can selectively elicit high rates of neural responding (Keidel, Keidel, \& Wigand, 1961: Kuffler, 1953). and are apparently important in eliciting attention responses (Sokolov, 1960; Teichner, Arees, \& Reilley, 1963; Treisman, 1964). When the stimulus is a complex sound and it is interrupted in a periodic manner, the interruption process can elicit the perception of pitch, which is independent of the spectral composition of the auditory stimulus (Miller \& Taylor, 1948: Schouten, 1940: Flanagan \& Guttman, 1960). These data would seem to indicate processing of certain aspects of the stimulus change or envelope, in addition to the spectral properties of the stimulus. Furthermore, this periodicity pitch can occur in the presence of a continuous. low-level masking noise (Harris, 1963), although with periodic pulses as stimuli the perceived pitch may be altered by the presence of the masking noise (Rosenberg, 1965).

Licklider $(1951,1959)$ hypothesized that the pitch of periodically interrupted noise stimuli is extracted by a process that is equivalent to an unnormalized short-term running autocorrelation in which the neural representation of the input waveform is temporally weighted by a decreasing exponential time function. Ritsma (1970), in discussing pulsed and repetition noise waveforms, proposed that a spectral analysis of the input waveform is performed by the basilar membrane, and that the activity in one "dominant" region of the membrane is subjected to an autocorrelation analysis which extracts the time interval between two pronounced positive peaks in the fine structure of the waveform. In addition to a simple spectral analysis of

*This research was supported by Grant NS 03856 to Central Institute for the Deaf and Fellowship NS 45299 to the author. both from the National Institute of Neurological Diseases and Stroke. The author is indebted to D. Eldredge. D. Ronken, R. Sorkin, and $C$. Watson for their helpful criticisms in the preparation of this paper.

$\div$ Current address: Department of Psychology. State Liniversity of New York at Binghamton. Binghamton. New York 13901. the waveform, both of these researchers propose an analysis which extracts periodicity information from the input signal.

The detectability of interrupted band-limited noise stimuli masked by a continuous, independently generated noise was investigated as a function of the average rate and the nature of the interruption process. A periodically interrupted, band-limited noise signal has essentially the same power spectrum as an equivalent band-limited noise which is aperiodically (randomly) interrupted. However, the aperiodically interrupted noise signal lacks the periodic envelope and the secondary peaks in any autocorrelation analysis which would be present for the periodically interrupted noise signal. If the auditory system selectively processes stimulus change, then interrupted noise signals should be more detectable than continuous noise signals of equal bandwidth, duration, and average power. Furthermore, if the auditory system is extracting periodicity information from the waveform envelope, periodically interrupted noise signals should be more detectable than equivalent aperiodically interrupted noise signals that have the same average interruption rate. However, if the interruption of the signal is processed only when the signal is clearly detectable, the periodically and the randomly interrupted signals should be equally detectable. Equal detection performance for the aperiodically interrupted and the continuous noise signals would indicate that either there is no special significance to the interruption process and the system temporally averages (integrates) the power (or power $/ \mathrm{Hz}$ ) in the signals or there is a partial temporal integration which is offset by the presence of the interruption extraction process. The detection hierarchy for such interrupted and continuous noise signals was determined.

Following the above arguments, the use of stimulus information provided by an interruption process in improving the detectability of the stimulus should be enhanced by exact knowledge of the temporal course of the waveform. Such frequency (i.e., $\tau$ or temporal delay) and synchronization information could be provided by a cueing signal presented to the nonstimulus ear. In 


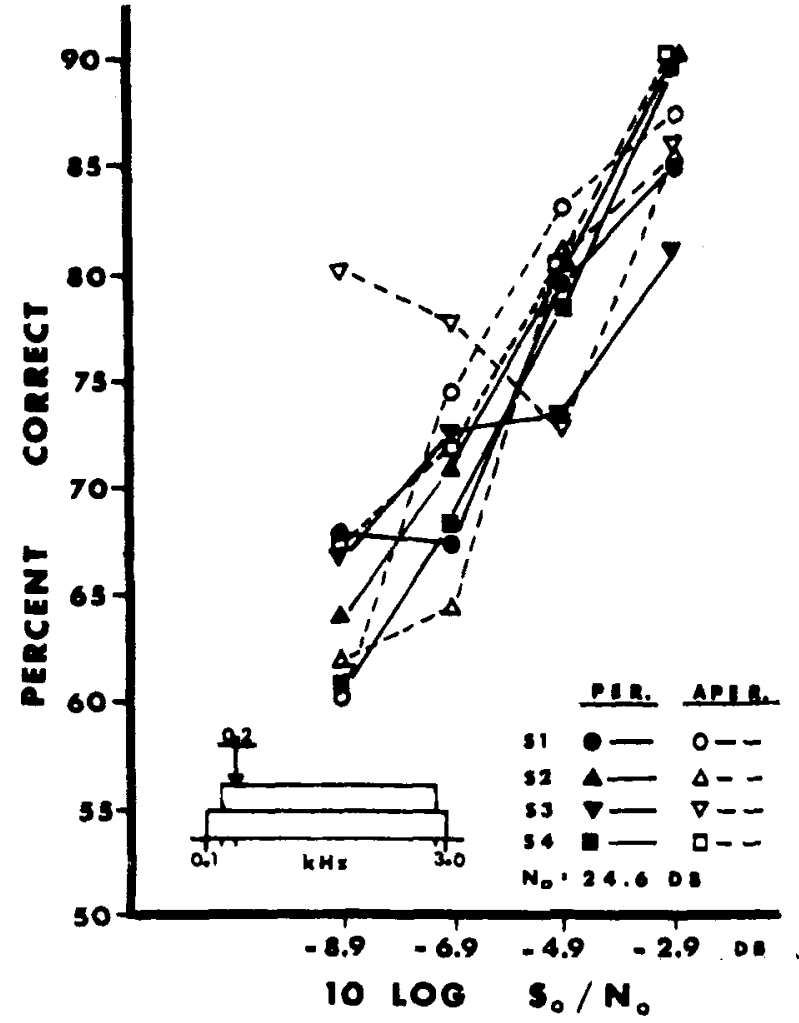

Fig. 1. Psychometric functions for the four Os at the average interruption rate of 200 ips $(0.2 \mathrm{kHz})$ for the periodic (filled symbols) and the aperiodic interruption process.

experiments with sinusoidal stimuli, such $\mathrm{MDCC}$ (monaural detection with contralateral cue) conditions have been shown to enhance the efficiency of the human $\mathrm{O}$ over simple monaural detection conditions (i.e., Sorkin, 1965; Taylor \& Forbes, 1969). In the context of the detection experiments described above, the contralateral cue should be an independently generated noise $\left(\mathrm{N}_{u}\right)$ interrupted synchronously with the actual or potential stimulus. This procedure avoids the confounding of lateralization effects. If there is an envelope or autocorrelation analysis, this unmasked contralateral signal should provide precise information about the envelope characteristics of the masked signal.

\section{METHOD}

The Os were four male high school students with clinically normal hearing who were employed for the study. The signal was a broadband noise, interrupted by Grason-Stadler electronic switches under the control of a PDP-8 computer. One electronic switch interrupted the noise signal in the predetermined periodic or aperiodic manner. The second switch determined which of the two temporally defined observation intervals contained the signal. On each trial, one of four stimulus intensities, determined by a computer-controlled attenuator, was employed. In this manner, a 4-point psychometric function was determined with each earphone placement. The signal was masked by a continuous, independently generated noise whose bandwidth ( 100 to $3.000 \mathrm{~Hz}$ ) exceeded that of the signal. The signal was interrupted by either a periodic sequence or one randomly selected from eight predetermined aperiodic sequences. All interruption sequences were matched in terms of both the average number of interruptions and the total signal power.

A monaural, two-interval, temporal forced-choice detection paradigm was employed in all conditions except the experiment requiring the $\mathrm{O}$ to discriminate the two types of interruption processes (Condition IId in Table 1 below). A same-different paradigm was employed in this discrimination experiment. In the control condition (Condition I in Table 1), the signal was uninterrupted. In the other conditions, the signal was interrupted at an average rate of 200,100 , or 50 interruptions per second (ips). These signals were 250,500 , or $1,000 \mathrm{msec}$ in duration, respectively (Conditions II, III, IV). All interruption sequendes had a $50 \%$ average duty cycle with exactly 50 interruptions. In the MDCC conditions (IIe, IIIb), the contralateral cue was an independent noise that was interrupted synchronously with, and had the same bandwidth as, the signal. The cue was presented in both observation intervals orthogonal to the presence of a signal in that interval. Two levels of the contralateral cue were employed. The two spectrum levels of the cue were 20.6 and $22.6 \mathrm{~dB}$ re 0.0002 microbar. The spectrum level of the monaural masking noise was either 24.6 or $31.6 \mathrm{~dB}$ re 0.0002 microbar. The signal bandwidth was 150 to $3.000 \mathrm{~Hz}$. except in one condition (IIc), where the lower limit was at $300 \mathrm{~Hz}$.

The Os were extensively practiced under all conditions until stable $d^{\prime}$ measures were obtained. During any block of 100 trials, the type (periodic vs random) and the rate of interruption were held constant. Initially, during any one 6-8-block session, both the rate and type of interruption process were fixed. Later, the type of interruption process was counterbalanced within a session with no measurable change in the d' measures. Following the practice sessions, 1,000 to 3,000 judgments were made by each $\mathrm{O}$ under each condition.

\section{RESULTS AND DISCUSSION}

The psychometric functions for the four Os under one of the 10 experimental conditions are shown in Fig. 1. The experimental conditions are outlined in the insert and indicate that the signal, whose bandwidth $(0.15-2.4 \mathrm{kHz})$ was narrower than the masking noise $(0.10-3.0 \mathrm{kHz})$, was interrupted at an average rate of 200 ips. The data for the periodic and aperiodic signals are highly similar. Data for the eight aperiodic sequences were equivalent, and thus were pooled.

The psychometric function for each $O$ under each condition was plotted by means of a least-squares linear regression of $\log \mathrm{d}^{\prime}$ vs $10 \log \left(\mathrm{S}_{\mathrm{o}} / \mathrm{N}_{\mathrm{o}}\right)$. The values of $10 \log \left(\mathrm{S}_{\mathrm{o}} / \mathrm{N}_{\mathrm{o}}\right)$ at $\mathrm{d}^{\prime}=1.0$ were determined and averaged across Os. The mean value of $10 \log \left(\mathrm{S}_{\mathrm{o}} / \mathrm{N}_{\mathrm{o}}\right)$ as well as the standard deviation under each condition may be found in Table 1. The data from Fig. 1 are under Condition IIa.

There were no significant differences between the periodically and aperiodically interrupted signals at any average interruption rate (see Table 1 , Items IIa, b, c; IIIa, b; IVa), at either noise level (IIa, b) or at either signal bandwidth (IIa, c). The interrupted signals (IIa) and the equivalent uninterrupted signal (Ia) were equal in detectability only at a level approximately $3 \mathrm{~dB}$ above the latter. This is consistent with the $3-\mathrm{dB}$ difference in total signal power due to the $50 \%$ duty cycle of the interruption process. These values of $10 \log \left(\mathrm{S}_{\mathrm{o}} / \mathrm{N}_{\mathrm{o}}\right)$ for 
Table 1

Mean Values of $10 \mathrm{Log}\left(\mathrm{S}_{\mathrm{o}} / \mathrm{N}_{\mathrm{o}}\right)$ Required for a Fixed Level of Performance $\left(\mathrm{d}^{\prime}=1.00\right)$ Under All Experimental Conditions

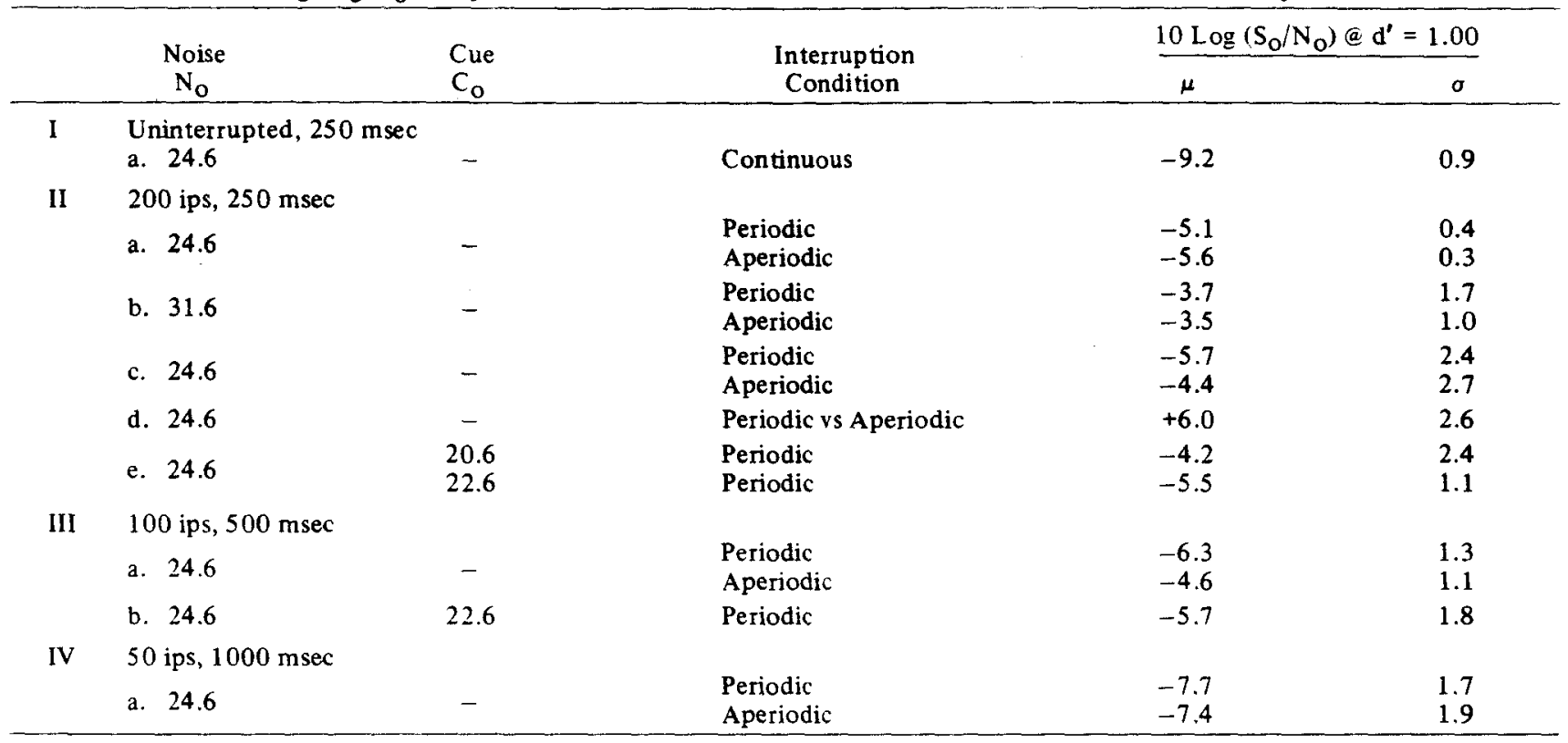

the uninterrupted noise signal are consistent with the values reported by Green (1960). Finally, the presence of the contralateral cue (IIe, f; IIIb) did not significantly affect the detectability of the periodically interrupted signal (IIa: IIIa), even after extensive practice. Furthermore, the masked periodically and aperiodically interrupted noise signals (IId) were discriminable $\left(\mathrm{d}^{\prime}=1.0\right)$ only at levels $10-12 \mathrm{~dB}$ above the level required for equivalent detection performance. ${ }^{1}$ This difference in the levels required for detection and discrimination is equivalent to the difference between the thresholds of detection and of intelligibility of speech (Hawkins \& Stevens, 1950).

In summary, it was found that: (1) interrupting a signal in either a periodic or an aperiodic manner does not appear to alter the detectability of the noise signal relative to an equivalent uninterrupted noise signal; (2) the presence of precise information about the interruption process in terms of a contralateral cue did not significantly affect the detectability of the interrupted signal: (3) like speech stimuli, the periodically and the aperiodically interrupted noise signals were discriminable only at levels $10-12 \mathrm{~dB}$ above the level required for detection. It would seem that, other than reducing total signal power, the interruption of a noise signal in either a periodic or a random manner did not alter the detectability of that masked noise signal, at least under the various conditions investigated.

\section{REFERENCES}

Flanagan, J. L., \& Guttman, N. On the pitch of periodic pulses. Journal of the Acoustical Society of America, 1960, 32, 1308-1319.

Green, D. M. Auditory detection of a noise signal. Journal of the
Acoustical Society of America, 1960, 32, 121-131.

Harris, G. G. Periodicity perception by using gated noise. Journal of the Acoustical Society of America, 1963, 35, 1229-1233.

Hawkins, J. E.. Jr., \& Stevens, S. S. The masking of pure tones and of speech by white noise. Journal of the Acoustical Society of America, 1950, 22, 6-13.

Keidel. W. D., Keidel, U. O., \& Wigand, M. E. Adaption: Loss or gain of sensory information? In W. A. Rosenblith (Ed.), Sensory communication. Cambridge: M.I.T. Press, 1961.

Kuffler, S. W. Discharge patterns and functional organization of mammalian retina. Journal of Neurophysiology, 1953, 16. 37-68.

Licklider, J. C. R. A duplex theory of pitch perception. Experientia, 1951, 7, 128-134.

Licklider, J. C. R. Three auditory theories. In S. Koch (Ed.), Psychology: A study' of a science. I. New York: MoGraw-Hill, 1959.

Miller, G. A., \& Taylor, W. G. The perception of repeated bursts of noise. Journal of the Acoustical Society of America, 1948, 20, 171-182.

Ritsma, R. J. Pitch: Result of an auto-correlation process. Sixth International Congress of Acoustics, Tokyo, 1968, Paper A-5-5.

Ritsma, R. J. Periodicity detection. In R. Plomp and G. F. Smoorenburg, (Eds). Frequency analysis and periodicity detection in hearing. Leiden: Sijthoff, 1970.

Rosenberg, A. E. Effect of masking on the pitch of periodic pulses. Journal of the Acoustical Society of America. 1965. 38, 747-758.

Schouten, J. F. The perception of pitch. Philips Technical Review, 1940, 5, 286-294.

Schouten, J. F. The residue revisited. In R. Plomp and G. F. Smoorenburg (Eds.), Frequency analysis and periodicit. detection in hearing. Leiden: Sijthoff, 1970.

Sokolov, E. N. In M. A. B. Brazier (Ed.), The central nerrous system and behalior. Transactions of the Third Conference. New York: Josiah Macy. Jr.. Foundation. 1960.

Sorkin, R. D. Uncertain signal detection with simultaneous contralateral cues. Journal of the Acoustical Society of America. 1965.38. 207.212.

Taylor. M. M.. \& Forbes. S. M. Monaural detection with 
contralateral cues (MDCC) I. Better than energy detector performance by human observers. Journal of the Acoustical Society of America. 1969, 46, 1519-1526.

Teichner. W. H.. Arees. E., \& Reilley. R. Noise and human performance. a psychophysiological approach. Ergonomics, 1963.6. 83-97.

Treisman. A. Selective attention in man. British Medical Bulletin, 1964. 20. 12-16.

\section{NOTE}

1. Both the periodically and the aperiodically interrupted noise signals had an irregular or rough quality near the levels required for detection. However, this rough quality did not appear to affect the detectability of these signals relative to the uninterrupted noise signal. The regular or periodic quality of the periodically interrupted noise signal began to appear at levels required for discriminating the periodically and aperiodically interrupted noise signals.

(Received for publication June 11, 1973; revision received July 23,1973 .) 